\title{
Sudden Cardiac Arrest in a Young Patient with Severe Pectus Excavatum
}

\author{
Rayan Jo Rachwan, MD', Andrea K. Purpura, MD², Basil M. Kahwash, MD ${ }^{1}$ \\ ${ }^{1}$ Department of Medicine, Indiana University School of Medicine, Indianapolis, Indiana. \\ ${ }^{2}$ Department of Emergency Medicine, Indiana University School of Medicine, Indianapolis, \\ Indiana.
}

Authorship: Rayan Jo Rachwan and Basil M. Kahwash have contributed equally to this paper.

Manuscript type: Patient-Centered Focused Review.

Running title: Sudden Cardiac Arrest in Severe Pectus Excavatum

Keywords: Pectus excavatum; Sudden cardiac arrest; Ventricular fibrillation; Implantable cardioverter defibrillator.

Funding Sources: None.

Conflict of Interest Statement: None declared.

*Corresponding Author:

Rayan Jo Rachwan, MD

Department of Medicine, Indiana University School of Medicine, Indianapolis, IN 46202, USA

Mailing Address: 700 North Alabama Street, Indianapolis, IN 46204

Email: rrachwan@iu.edu

This is the author's manuscript of the article published in final edited form as:

Rachwan, R. J., Purpura, A. K., \& Kahwash, B. M. (2018). Sudden Cardiac Arrest in a Young Patient with Severe Pectus Excavatum,. The American Journal of the Medical Sciences. https://doi.org/10.1016/j.amjms.2018.06.002 


\section{$\underline{\text { Abstract }}$}

We report a case of sudden cardiac arrest in the setting of ventricular fibrillation in a previously healthy 19-year-old male. Chest imaging demonstrated severe pectus excavatum with Pectus Severity Index of 22.7. Extensive workup was unrevealing for other cardiopulmonary etiologies, including conduction and structural abnormalities. The patient was scheduled for a Ravitch procedure and was discharged on a wearable defibrillator vest for temporary protection against ventricular arrhythmias. Later, the patient underwent subcutaneous implantable cardioverter defibrillator placement. Sudden cardiac arrest as an initial presentation of pectus excavatum is a rare entity scarcely discussed in medical literature. In this patient-centered focused review, we explore this unique case and offer our management approach amid the lack of concrete guidelines.

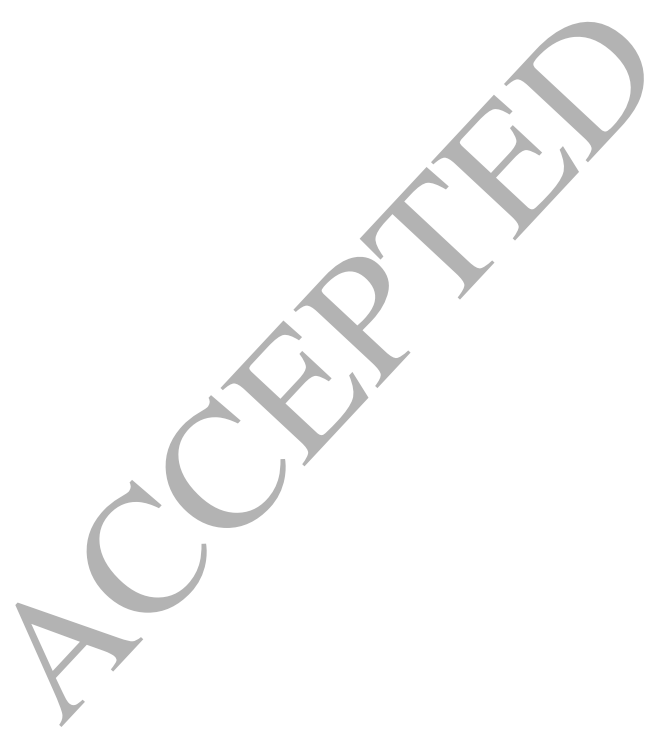




\section{$\underline{\text { Introduction }}$}

Pectus excavatum $(\mathrm{PE})$ is a congenital abnormality with unknown etiology characterized by lower end sternal depression, with or without rib protrusion. PE is the most common congenital chest wall deformity, accounting for $90 \%$ of such cases. It occurs in 1 of $400-1000$ births and is 3-5 times more prevalent in males. ${ }^{1} \mathrm{PE}$ deformity is commonly, though not always, associated with rare connective tissue diseases such as Marfan syndrome, Poland syndrome and EhlerDanlos syndrome. When symptomatic, patients may present with cardiac or pulmonary complaints including shortness of breath, exercise intolerance, and syncope. In rare cases, arrhythmias have been attributed to $\mathrm{PE}$, and are believed to originate from direct sternal impingement of the myocardium. , $^{2}$

Sudden cardiac arrest (SCA) in the setting of ventricular fibrillation (VF) as an initial presentation of symptomatic $\mathrm{PE}$ is a rare entity with scarce literature. We hereby present a case of VF-induced SCA in a young patient with severe PE with no previous cardiopulmonary symptoms, and describe the diagnostic and therapeutic modalities used in his treatment. 


\section{Case Presentation}

A 19-year-old previously healthy male presented to the emergency department after SCA. He collapsed at home, and emergency response personnel found him to be in VF. He was successfully resuscitated after 1 cycle of cardiopulmonary resuscitation followed by 1 shock from an automated external defibrillator, and subsequently transferred to a nearby hospital. Electrocardiogram (ECG) in the emergency department showed an entirely negative $P$ wave in lead V1, inverted T waves in leads V1-V2 and incomplete right bundle branch block (Figure 1).

The corrected QT interval was within normal limits. The patient was intubated and admitted to the intensive care unit for post-cardiac arrest treatment, and the hypothermia protocol was initiated. Physical exam was unremarkable except for PE (Figure 2). Laboratory evaluation revealed a normal complete blood count and normal electrolytes. His troponin level peaked at $1.4 \mathrm{ng} / \mathrm{ml}$ and he had a negative urine drug screen. After clinical stabilization, he was extubated and had no further symptoms or significant events on telemetry monitoring.

The patient's initial cardiac arrest workup included a chest x-ray, which demonstrated steep angulation of the anterior ribs with loss of the right heart border on posteroanterior view and posterior displacement of the sternum on lateral view; but was otherwise unrevealing for acute cardiopulmonary process. Chest computed tomography (CT) showed compression of the heart by severe sternal deformity resulting in ballooning of right ventricular (RV) apex with leftward shift. Chest CT findings were later confirmed by cardiac magnetic resonance imaging (MRI), which did not show myocardial scarring or evidence of arrhythmogenic RV dysplasia (Figure 3). Thoracic width-to-depth ratio (Pectus Severity Index or PSI) was found to be 22.7 (normal 
$<2.5$ ). He further underwent CT angiography of the coronary arteries, which did not show any arterial pathology. Transthoracic echocardiogram (TTE) was interpreted as having normal left and right ventricular size and contractility. Procainamide provocation test ruled out Brugada syndrome. An electrophysiology study with programmed ventricular stimulation was performed and showed inducible VF with triple ventricular extrastimuli at the RV apex. This response was interpreted as nonspecific and no target site could be isolated for catheter ablation. The patient was scheduled for operative correction of the defect using the Ravitch procedure, and was recommended to wear a defibrillator vest while awaiting implantable cardioverter defibrillator (ICD) placement and allowing surgical wounds to heal. In addition, he received genetic counseling and underwent genetic testing for hereditary arrhythmia. Results showed no identifiable pathologic variants associated with arrhythmia. Three months after surgical repair, the patient underwent subcutaneous ICD placement. ICD interrogation at the 1-month and 2month follow-ups did not record any arrhythmogenic events. 


\section{Discussion}

The clinical sequelae of PE can vary from cosmetic anxiety to cardiopulmonary compromise. Cardiopulmonary symptoms most commonly manifest as exercise intolerance (82\%), followed by chest pain (68\%), poor endurance $(67 \%)$ and shortness of breath $(42 \%))^{2,3}$ Although less common, RV compression by PE can lead to conduction abnormalities that include atrial fibrillation and supraventricular tachycardia. ${ }^{4-6} \mathrm{~A}$ leftward deviation of the heart may occur due to sternal compression.

Commonly observed ECG patterns in PE include right axis deviation, persistent juvenile T-wave pattern (T wave inversion in lead V1-3) and incomplete right bundle branch block. One less common pattern, a predominantly or entirely negative $\mathrm{P}$ wave, can be observed in right precordial leads (especially lead V1) ${ }^{6,7}$ These patterns have been attributed to vectoral changes, particularly in the precordial leads, related to cardiac compression and leftward displacement. With the exception of right axis deviation, these findings were noted on our patient's ECG.

TTE is an essential diagnostic tool in patients presenting with cardiopulmonary symptoms. In cases of PE, TTE can be technically challenging due to chest wall deformity that may obstruct normal thoracic window views. ${ }^{8}$ Such obstruction mandates modified use of the three standard windows (apical, parasternal and subcostal), or a more invasive diagnostic test such as transesophageal echocardiography. TTE can be helpful in detecting structural abnormalities related to $\mathrm{PE}$, ranging from subtle RV wall motion abnormalities to gross $\mathrm{RV}$ compression, which 
would be an indication for surgical repair. There were no significant functional or structural abnormalities found in our patient on TTE.

Three dimensional imaging, usually chest CT or cardiac MRI, is needed for the stratification and anticipated management of PE. The PSI (also known as the Haller Index) is obtained from these images, and is determined by calculating the ratio of the maximal lateral diameter of the inside of the ribcage to the shortest distance between the sternum and the anterior surface of the spine, at the distal third of the sternum. ${ }^{9} \mathrm{~A}$ PSI greater than 3.25 is considered severe. Although there are reports that state that the severity of the index does not necessarily correlate with symptoms, ${ }^{8}$ our patient's PSI of 22.7 is significantly high and may have contributed to cardiac arrhythmia. PSI of this magnitude has not been evaluated as to its cardiopulmonary impact.

Expert opinion recommends surgical correction in PE patients with at least 2 of the following 4 criteria: progressive deformity with associated cardiorespiratory symptoms (e.g. exercise intolerance or chest pain); PSI of 3.25 or more and imaging that demonstrates cardiac compression or displacement; abnormal pulmonary function studies consistent with either restrictive or obstructive disease; and cardiac evaluation documenting compression or displacement, or other pathological findings such as arrhythmia or mitral valve prolapse. ${ }^{9}$ Our patient met the need for surgery by satisfying 3 of the 4 criteria.

Surgical approaches usually vary based on the practitioner's preference. The two most common procedures are the Ravitch technique, which involves open sternotomy and cartilage resection, and the less invasive Nuss procedure, in which a metal bar is placed retrosternally using a small lateral incision. In our case, the surgeon opted for the Ravitch procedure. 
SCA occurs at an annual incidence of $1.3-8$ cases per 100,000 persons in the pediatric and adolescent population. ${ }^{10}$ Currently, there is no consensus on the management of SCA in the setting of PE. A variety of treatment modalities for symptomatic ventricular arrhythmias in patients with PE have been described by other case reports. ${ }^{5,11-13}$ Hamoud et al ${ }^{11}$ describe the occurrence of SCA due to VF in a previously asymptomatic subject with PE who underwent subcutaneous ICD placement without prior corrective surgery. DeSimone et al ${ }^{12}$ reported a case of sustained and nonsustained monomorphic ventricular tachycardia (VT) in a patient with PE. A catheter ablation was performed followed by surgical repair due to concern of possible residual arrhythmogenic foci from $\mathrm{RV}$ compression. A repeat electrophysiology study was performed at 1-year follow-up and obtained normal results. The 2-year follow-up did not show recurrence of VT. Still, whether resolution of the arrhythmia was attributable to catheter ablation or surgical repair is unclear. Chan Wah Hak et al ${ }^{5}$ reported surgical repair of PE on a patient with sustained monomorphic VT on exertion, with no recurrence of VT upon follow-up at 3 years. Similarly, Pimenta et al ${ }^{13}$ explored the case of a 14-year-old boy with palpitations from polymorphic VT who underwent a Nuss procedure, with no recurrence of symptoms after 2 years.

Current guidelines recommend ICD placement in patients who survive a VT/VF arrest not due to reversible causes, and who have an expected survival of more than 1 year. ${ }^{14}$ In our patient, extensive workup that included multiple invasive and noninvasive modalities was performed and was unrevealing for any structural or electrical cardiac abnormalities, except for severe RV compression by the sternum. In this context, PE could have been the most plausible etiology of the patient's SCA. On the other hand, it is uncertain whether PE can be considered a reversible 
cause of SCA due to a lack of available data. Therefore, it was reasonable to proceed with ICD placement post-surgical repair as secondary prevention of SCA in our patient. We opted for the implantation of a subcutaneous rather than a transvenous ICD by reason of lower complication risk profile as well as lower risk of lead failure, especially in patients with active lifestyles. ${ }^{15-17}$ Furthermore, a young patient with a favorable long-term prognosis is more likely to require multiple device replacements, which are associated with higher mortality and morbidity in case of transvenous ICD. ${ }^{17,18}$ The patient wore a defibrillator vest as a bridge to ICD placement. 


\section{Conclusions}

Life-threatening arrhythmia is a rare manifestation of PE. Expert opinion recommends surgical correction of the PE deformity if criteria are met. Extensive workup in our patient's case suggests that cardiac arrest likely occurred in relation to his PE; however, a cause-and-effect relationship has not been firmly established. The absence of ventricular arrhythmias at longterm follow up after repair would lend support to the hypothesis of PE-induced arrest. A consensus on ICD placement following SCA after or in place of operative repair has not yet been established. Therefore, we suggest applying the standard ICD guidelines for secondary prevention of SCA, awaiting further data that would explore PE as a reversible cause of SCA. 


\section{$\underline{\text { References }}$}

1. Fokin AA, Steuerwald NM, Ahrens WA, et al. Anatomical, histologic, and genetic characteristics of congenital chest wall deformities. InSeminars in thoracic and cardiovascular surgery 2009 May 1 (Vol. 21, No. 1, pp. 44-57).

2. Bay V, Farthmann E, Naegele U. Unoperated funnel chest in middle and advances age: evaluation of indications for operation. J Pediatr Surg 1970; 5:606.

3. Nuss D, Kelly RE Jr. Minimally invasive surgical correction of chest wall deformities in children (Nuss procedure). Adv Pediatr 2008; 55:395.

4. Tran NT, Klein JL, Mounsey JP, et al. Lone atrial fibrillation is associated with pectus excavatum. Heart Rhythm. 2013 Sep 30;10(9):1263-9.

5. Chan Wah Hak YS, Lim YP, Liew R, et al. Pectus excavatum: uncommon electrical abnormalities caused by extrinsic right ventricular compression. Journal of cardiovascular electrophysiology. 2014 Mar 1;25(3):324-7.

6. Reusch CS. Hemodynamic studies in pectus excavatum. Circulation. 1961 Nov $1 ; 24(5): 1143-50$

7. de Oliveira JM, Sambhi MP, Zimmerman HA. The electrocardiogram in pectus excavatum. British heart journal. 1958 Oct;20(4):495.

8. Jaroszewski DE, Warsame TA, Chandrasekaran K, et al. Right ventricular compression observed in echocardiography from pectus excavatum deformity. Journal of cardiovascular ultrasound. 2011 Dec 1;19(4):192-5.

9. Nuss D, Obermeyer RJ, Kelly Jr RE. Pectus excavatum from a pediatric surgeon's perspective. Annals of cardiothoracic surgery. 2016 Sep;5(5):493. 
10. Shen WK, Edwards WD, Hammill SC, et al. Sudden unexpected nontraumatic death in 54 young adults: a 30-year population-based study. The American journal of cardiology. 1995 Jul 15;76(3):148-52.

11. Hamoud A, Barry J. Subcutaneous implantable cardioverter defibrillator in a patient with pectus excavatum. Pacing and Clinical Electrophysiology. 2013 May 1;36(5).

12. DeSimone CV, Sagar S, Moir C, et al. Combined surgical and ablative cure for localized sternal compression-induced cardiomyopathy and ventricular tachyarrhythmia. The Journal of thoracic and cardiovascular surgery. 2012 Sep 1;144(3):e85-7.

13. Pimenta J, Vieira A, Henriques-Coelho T. Ventricular arrhythmia solved by surgical correction of pectus excavatum. Interactive cardiovascular and thoracic surgery. 2017 Dec 12.

14. Al-Khatib SM, Stevenson WG, Ackerman MJ, et al. 2017 AHA/ACC/HRS Guideline for Management of Patients With Ventricular Arrhythmias and the Prevention of Sudden Cardiac Death: A Report of the American College of Cardiology/American Heart Association Task Force on Clinical Practice Guidelines and the Heart Rhythm Society. Journal of the American College of Cardiology. 2017 Oct 30:24390.

15. Bogush N, Espinosa RE, Cannon BC, et al. Selecting the right defibrillator in the younger patient: Transvenous, epicardial or subcutaneous?. International journal of cardiology. $2018 \operatorname{Jan} 1 ; 250: 133-8$.

16. Ben Morrison T, Rea RF, Hodge DO, et al. Risk factors for implantable defibrillator lead fracture in a recalled and a nonrecalled lead. Journal of cardiovascular electrophysiology. 2010 Jun 1;21(6):671-7. 
17. Bardy GH, Smith WM, Hood MA, et al. An entirely subcutaneous implantable cardioverter-defibrillator. New England Journal of Medicine. 2010 Jul 1;363(1):36-44.

18. Deshmukh A, Patel N, Noseworthy PA, et al. Trends in Utilization and Adverse Outcomes Associated with Transvenous Lead Removal in the United States. Circulation. 2015 Nov 3:CIRCULATIONAHA-114. 


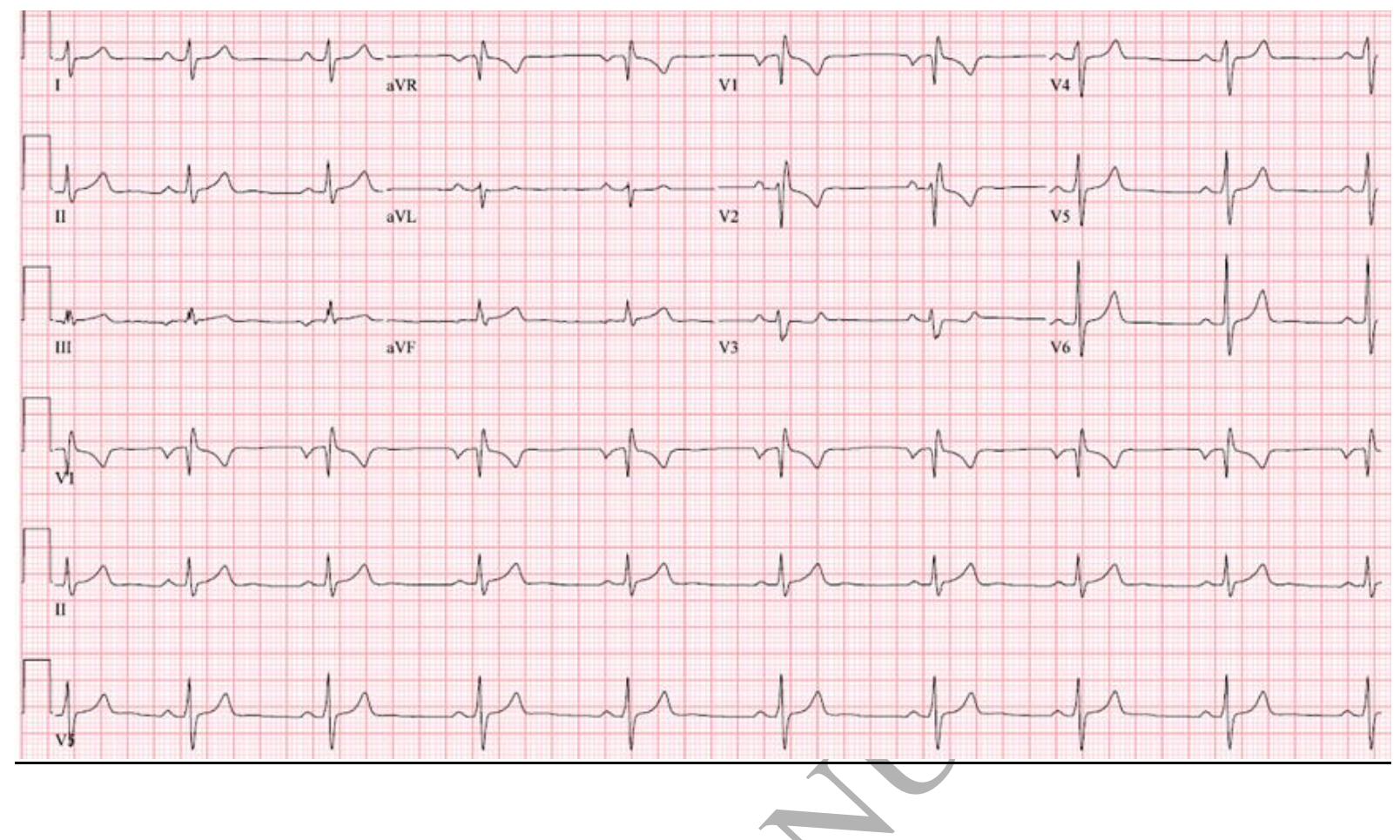

Figure 1: Electrocardiogram showing sinus bradycardia with incomplete right bundle branch block, in addition to an entirely negative $\mathrm{P}$ wave in lead V1.

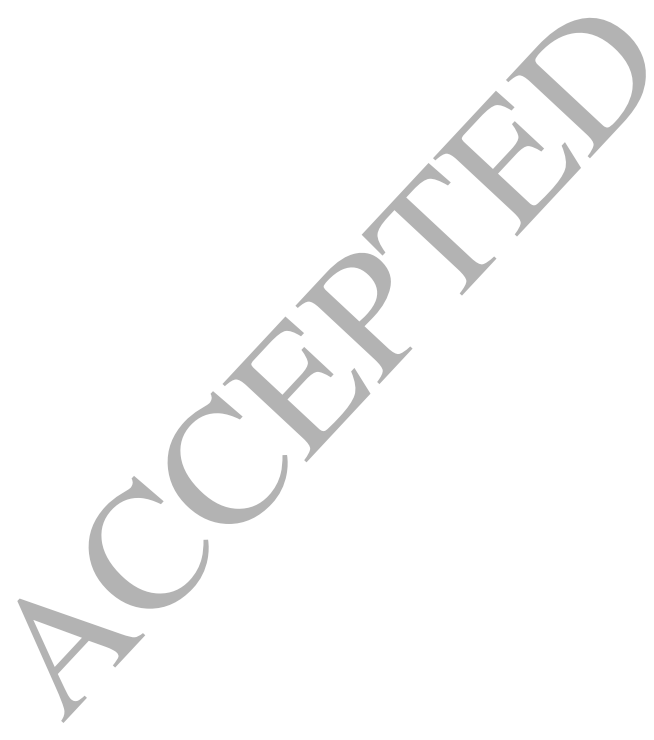




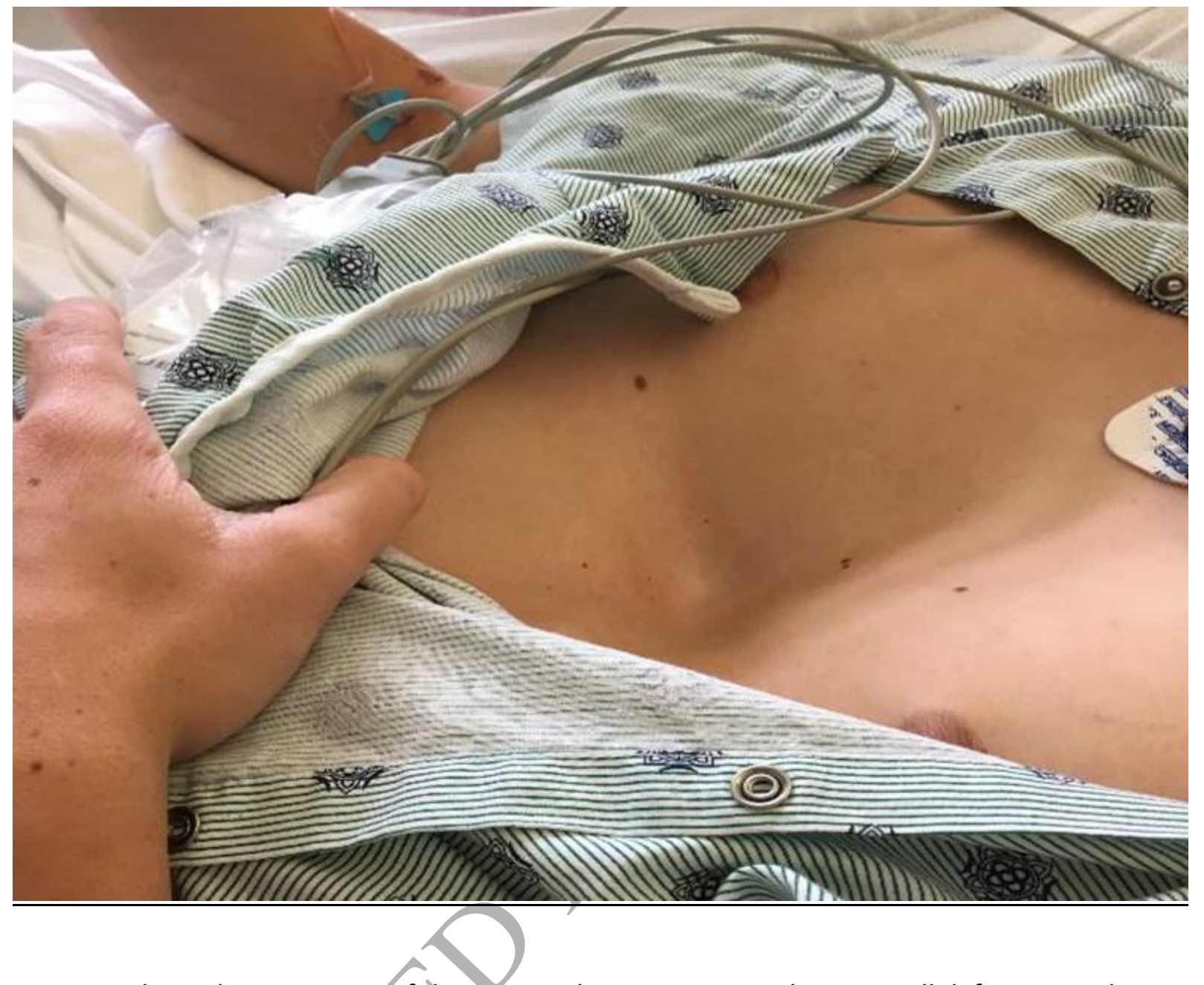

Figure 2: Physical examination of the patient showing anterior thoracic wall deformity with depression of the sternum.

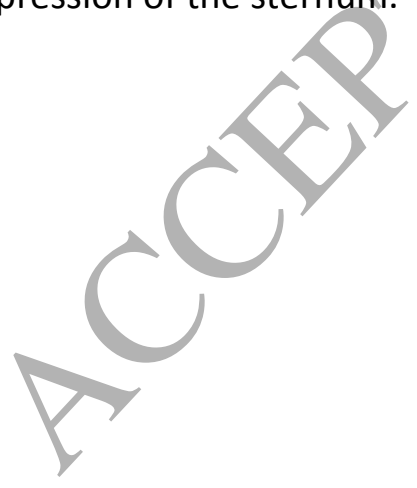




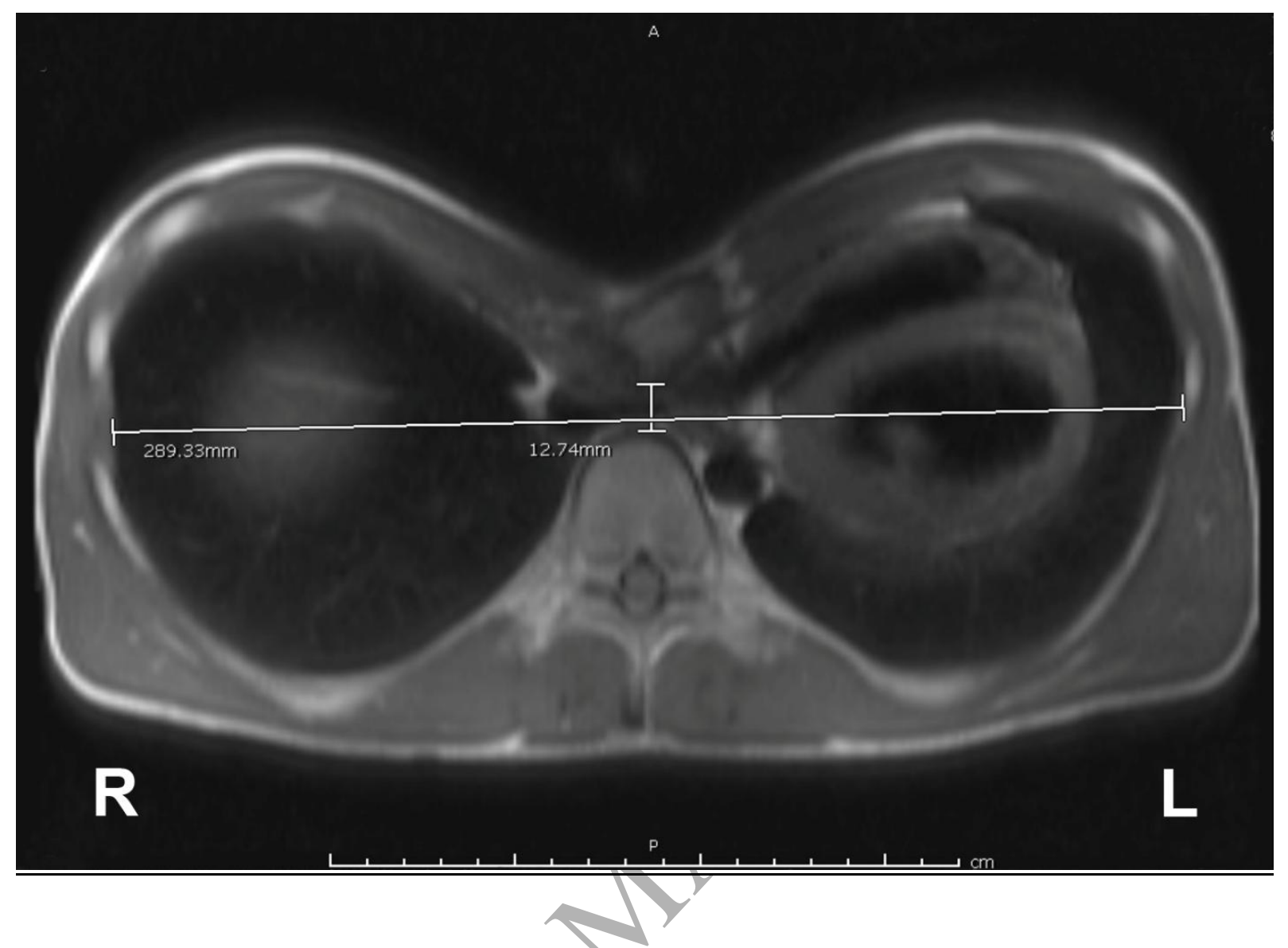

Figure 3: Cardiac magnetic resonance imaging showing compression of the heart and ballooning of right ventricular apex with leftward shift. Thoracic width-to-depth ratio (Pectus Severity Index) calculated as 22.7 at the distal third of the sternum.

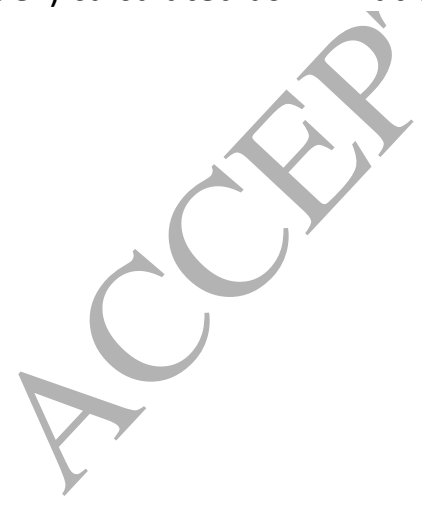

